\title{
Design and Implementation of the Intelligent and Interactive Task Allocation System
}

\author{
YU Jing-Jing ${ }^{1, a}$, PAN Jing-Chang ${ }^{2, b_{*}}$ \\ ${ }^{1,2}$ Shandong University, Weihai. 264209, China

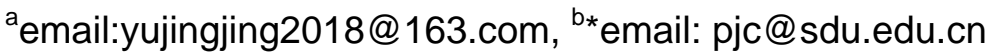

Keywords: Task Allocation; Greedy Algorithm; Progress Check

\begin{abstract}
TA (Task Allocation) system is designed for the workers of National Astronomical Observatory, Chinese Academy of Sciences. TA plays an important role in simplifying the manual operation, improving work efficiency and reducing man-made mistakes. TA achieves an intellectualized even distribution for the task of data processing and it proposes an interactive way of task allocation. The result of automatic allocation can be fine-tuned by drag-and-drop so it realizes convenient human interaction. This paper discusses the design and implementation of the intelligent and interactive task allocation system in detail and worked out an optimistic algorithm of intelligent task allocation.
\end{abstract}

\section{Introduction}

Astronomical observation data are processed in unit of sky regions (short for sky), and the information of each sky contains the sky number, observation date, and spectrum number. Before the design of TA, all the tasks above need manual operation. Because of the huge amount of data and the differences in the number of spectrum in each sky, there are so many difficulties to manual operation. TA is to assign a great deal of spectrums to several staffs to process and analyze. TA uses the sky or spectrometer as the basic unit in assignment, and intelligent assignment is on the principle of making the spectrums approximately equal to everyone and administrator can make adjustments to fine-tune the allocation result according to actual situation. With the goal of improving staffs' work efficiency and quality, TA solves these problems effectively. At the same time, TA achieves the goal of reviewing the progress in real-time of each person. This paper design an efficient and accurate task allocation algorithm in order to realize intelligent task allocation, and proposed an interactive allocation method to humanize the adjustment of the allocation result. This system is based on Java+hibernate+bootstrap+jquery technology in B/S architecture with MVC[1][2] model, a good solution with browser compatibility and platform-independence $[3][4][5][6]$.

\section{System Design}

There are three modules in TA system, TA-1D, TA-2D and Progress check 1D, as shown in Fig.1. TA-1D and TA-2D is designed to complete the task allocation of 1D and 2D stage, and the module Progress check 1D is for checking the progress of $1 \mathrm{D}$ spectrum checking. Among them, the procedure of TA-1D is similar to TA-2D. The only difference is the basic unit in allocation, TA-1D uses SR as the basic unit instead of spectrometer.

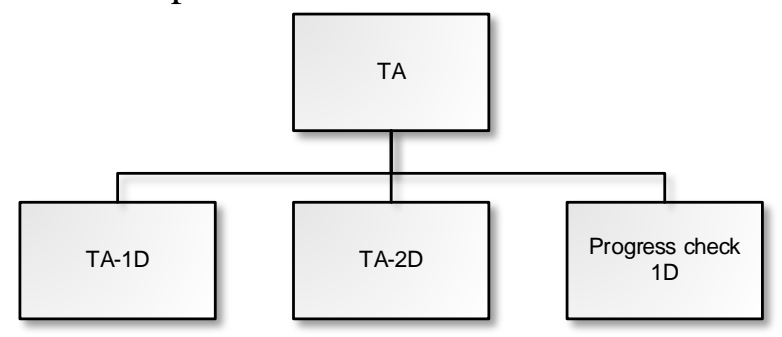

Fig.1. TA Structure 
The ordinary users do not have to sign in the system to view the allocation result, they can find it in the task_allocate_result file which is downloaded locally after the administrator submits the task allocation result.

\section{Process of Task Allocation System}

The procedure of TA-1D is similar to TA-2D, function design flow chart is shown in Fig.2. First, retrieve the suitable Pipeline version and date range from database. The $1 \mathrm{D}$ pipeline version can be obtained from table "spec_check" and 2D pipeline version can be obtained from table "obj_info" in database "dbregular2". Users choose the pipeline version, and choose or input the data range and number of people then click the button "pre-allocate" to make the system allocate the task in the data range automatically in background. When this process completed, the result would be shown in the view in the form of list. Administrator can fine-tune the allocation result according to actual situation by drag-and-drop. After the adjustment finished, click the button "submit allocation", user can get the table of allocation result in the view and the HTML file will be downloaded locally. The table of the allocation result contains the links that can link to the page which shows the detail information of tasks.

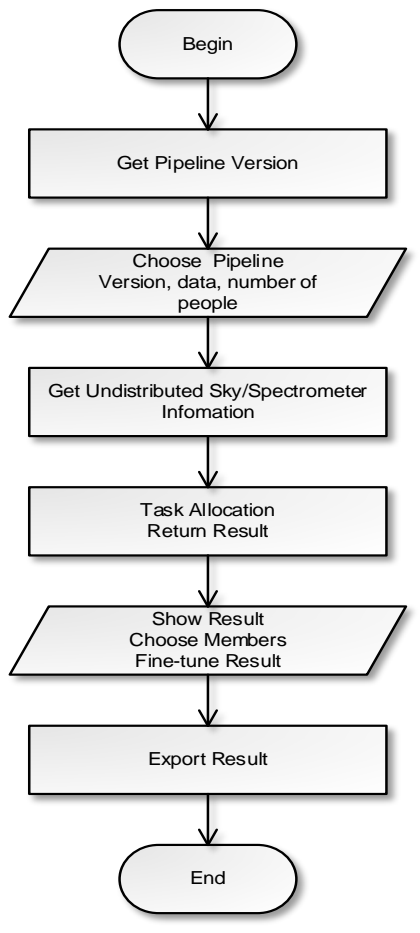

Fig.2. Function Design Flow Chart

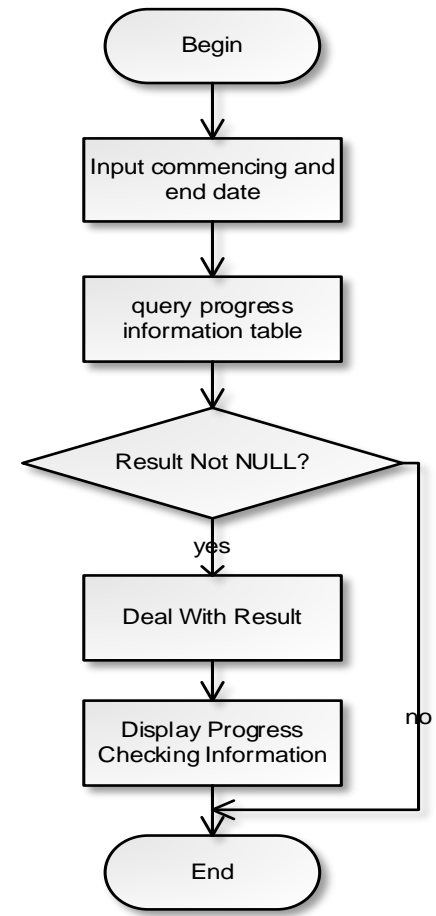

Fig.3. Progress Checking Flow Chart

Progress-Check-1D is designed to check the progress of 1D spectrum checking, and the checking result is shown in two forms, one is based on s-r and the other is based on member. According to different demands, managers can check the progress intuitively. The function of this module consists of four steps: input the commencing and end date for query, query progress information table, deal with result, display progress check information. The date selection box "start date" and "end date" is for the selection of checking date. After select the begin date and end date, users can start the operation of query progress information table. It checks the progress information table "checkstatus" and gets suitable records according to date range input above. The system will carry out the operation Deal-with-Result for the records which match the conditions, and put the results into a new table. A procedure called Display-Progress-Check-Information is designed to show the progress information treated, including sky based result and member based result. The function design flow chart of progress check 1D is shown in Fig.3. 


\section{System database design}

As a subsystem of the LAMOST Product Process Monitoring System, TA uses two databases, one is lasacv2 and the other is dbregular2, and there are eight tables altogether, as shown in Fig.4. They are member information table (t_member), unit information table (t_unit), role table (t_role), privilege table (t_privilege), role-privilege relationship table (t_role_privilege), progress check table (t_progress_check_result) in database “dbregular2” and object information table (obj_info), manual inspection table (spec_check) in database "lasacv2". Tables in database "lasacv2" are for getting the information of members and units, and others are for getting the information of tasks in details such as s-r and so on.

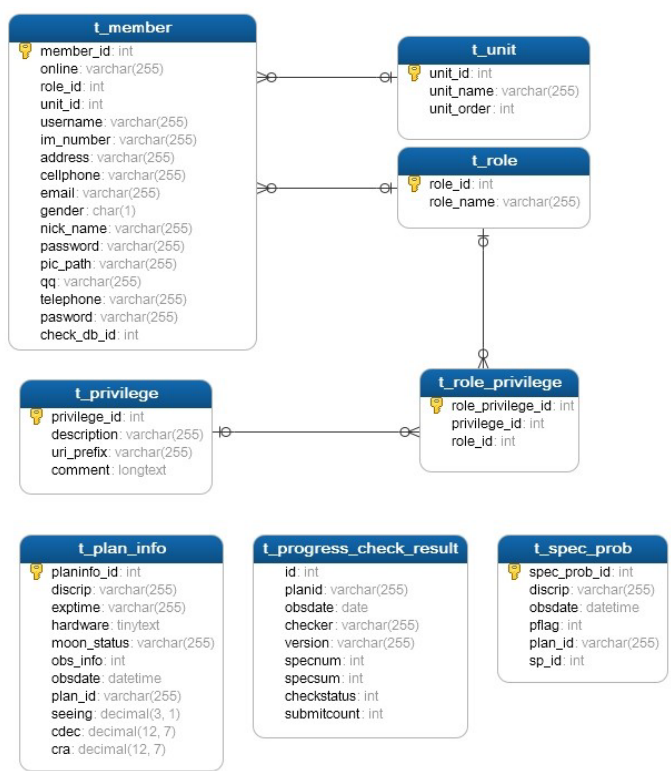

Fig.4. Database Design Diagram

\section{System Implementation}

The development of this system mainly uses java programing language, combining with bootstrap[7], hibernate, jQuery[8] and other related technology and frameworks, system implementation is mainly divided into TA-1D, TA-2D, Progress check $1 \mathrm{D}$, due to the similarity between TA-1D and TA-2D. For simplicity, we only introduce TA-1D.

\section{The Implementation of Task Allocation Algorithm}

The question need to be solved is that we need to get some skies from databases in a date range to assign to individuals. Daily weather conditions is changeable, which means that the number of spectrum in each sky often varies greatly, and we want to make the distribution average in the greatest degree. We used greedy algorithm[9] to achieve our goal. The core of the greedy algorithm is to make the best choice in the current situation. Suppose the number of people is $\mathrm{k}$. There are five steps to realize this algorithm. (1) get the skies in the date range and sort them from most to least according to the number of spectrum. (2) give a sky to each in turn, and give the next one to the one who gets the least number of spectrum. (3) sum up everyone's spectrum number, and get the guy with the minimum spectrum number. (4) give the next sky to the one we have got in the last step. (5) repeat last two steps until all the skies are assigned. We tested the time performance between the greedy algorithm and other two algorithms (Variance method and Exhaustive method) and found that the greedy algorithm used the minimum time. So we decided to use greedy algorithm to achieve the allocation. 


\section{The Implementation of Task Allocation Workflow}

When users enter the TA page, the system will gets the pipeline versions from databases and display them in the drop-down list box for user to select. In the page users select or input the date range and input the number of people and then click the button "pre-allocate", the parameters, pipeline version, data range and number of people, will be transferred to business logic handler in the background for the program to retrieve appropriate records. Then the system will call the method of TA to finish the distribution. After distribution, the result will be returned to the front page in the form of list. The task allocation result is shown as Fig.5.

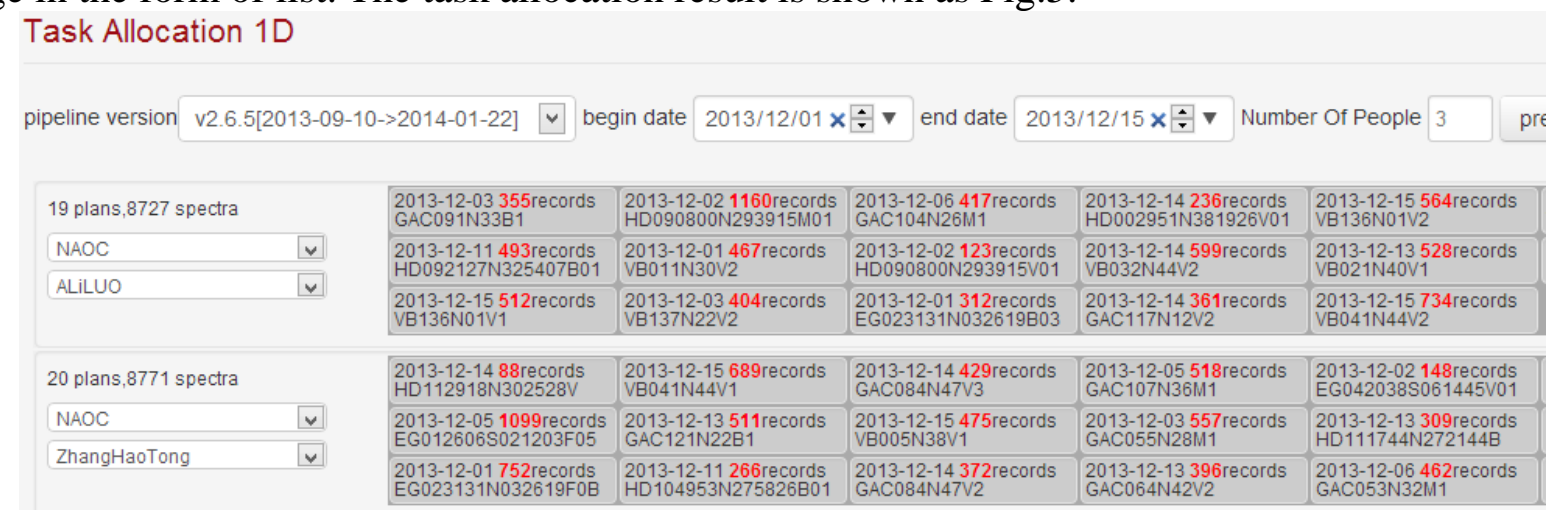

Fig.5. Task Allocation result

\section{Acknowledgement}

In this paper was sponsored by The Natural Science Foundation of China (Project No. U1431102). *Corresponding author: Jingchang Pan.

\section{References}

[1] Xu Sheng, Wu Chuan sheng, Chen Yue yang, Cui Lei. Application of MVC in Web System, Computer Technology and Application Progress, 2006, 953 956.

[2] Lu Rong xing, Yu Zhou, Ruan Yong liang, Wang Zhi qiang, Study and Implementation of MVC Design Pattern on J2EE, Application Research of Computer,2003,144 146.

[3] Shi Mei lin, Yang Guang xin, Xiang Yong, Wu Shang guang, WfMS: WORKFLOW MANAGEMENT SYSTEM. CHINESE J.COMPUTERS 1999.

[4] Luo Hai bin, Fan Yu Shun Wu Cheng, Reviewed of Workflow Technology, Journal of Software.2000,11(7):899 907.

[5] Zhao Wen, Hu Wen hui, Zhang Shi kun, Wang Li fu. Study and Application of Workflow Meta-Model, Journal of Software.2003.

[6] Xu Liang, Zhang Li, Fan Zhi qiang. An Approach of Real-Time Workflow Modeling Based on UML, Journal of Computer Research and Development, 2010, 1184 1191.

[7] Cornelia Györödi,Robert Györödi,George Pecherle,Tamas Lorand, Roșu Alin. Web 2.0 Technologies with jQuery and Ajax[J]. Journal of Computer Science and Control Systems,2009,2(2):11

[8] Jyotishka Datta,Jayanta K. Ghosh. Bootstrap-An exploration[J]. Statistical Methodology,2013.

[9] Vladimir N. Temlyakov,Pavel Zheltov. On performance of greedy algorithms[J]. Journal of Approximation Theory,2011,163(9): 1134 1145. 